\title{
PÓ-DE-ACIARIA COMO FONTE DE ZINCO PARA O MILHO E SEU EFEITO NA DISPONIBILIDADE DE METAIS PESADOS ${ }^{(1)}$
}

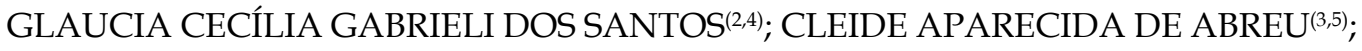 \\ OTÁVIO ANTONIO DE CAMARGO ${ }^{(3,5)}$; MÔNICA FERREIRA DE ABREU(3,5)
}

\begin{abstract}
RESUMO
O objetivo deste trabalho foi avaliar o pó-de-aciaria como fonte de zinco para o milho (Zea mays L.) e seu efeito no acúmulo e disponibilidade de $\mathrm{Cd}, \mathrm{Cr}, \mathrm{Ni}$ e $\mathrm{Pb}$ no solo e nas plantas. O experimento foi realizado em casa de vegetação, utilizando-se dois Latossolos Vermelhos e em dois valores de $\mathrm{pH}(5,0$ e 6,0). As fontes de zinco foram o pó-de-aciaria e o sulfato de zinco aplicados nas doses de 0, 5, 50 e 150 mg.dm ${ }^{-3}$ de $\mathrm{Zn}$. Fez-se a extração de $\mathrm{Zn}, \mathrm{Pb}, \mathrm{Cd}, \mathrm{Cr}$ e $\mathrm{Ni}$ do solo utilizando-se as soluções de DTPA e de Mehlich-1. A menor dose de Zn adicionada ao solo, nas duas fontes, proporcionou teores na parte aérea da planta superiores ao nível crítico para a cultura. As concentrações dos metais $\mathrm{Pb}, \mathrm{Cd}, \mathrm{Cr}$ e $\mathrm{Ni}$, tanto na parte aérea como no solo, foram menores que os níveis críticos para toxicidade. Os coeficientes de correlação entre o Zn extraído do solo pelas duas soluções e a concentração de Zn na parte aérea do milho foram significativos e semelhantes para ambos os solos. O mesmo não foi observado para os outros elementos. Conclui-se que, nas condições estudadas, o pó-de-aciaria é uma fonte eficiente de Zn para o milho; os extratores são adequados para determinar a disponibilidade desse elemento, ineficientes, entretanto, para os outros metais estudados. $\mathrm{O}$ pH alterou a disponibilidade do $\mathrm{Zn}$ diminuindo a disponibilidade com seu aumento. Palavras-chave: metais pesados, resíduo siderúrgico, extratores.
\end{abstract}

\section{ABSTRACT \\ FLUE DUST AS ZINC SOURCE TO CORN AND ITS EFFECT IN HEAVY METALS AVAILABILITY}

\begin{abstract}
The purpose of this study was to assess the effect of application of increasing doses of flue dust on the accumulation and availability of the metals $\mathrm{Zn}, \mathrm{Cd}, \mathrm{Cu}, \mathrm{Ni}$ and $\mathrm{Pb}$ in two surface soil samples and in corn plants (Zea mays L.). The experiment was carried out at the Instituto Agronômico de Campinas, São Paulo State, Brazil, under greenhouse conditions, using corn as test plant, grown in two Typic Eutrorthox at two pH levels $\left(5,0\right.$ e 6,0). The $\mathrm{Zn}$ sources were flue dust and pure $\mathrm{ZnSO}_{4}$ applied at the following rates: 0; 5; 50 and $150 \mathrm{mg} \cdot \mathrm{dm}^{-3} \mathrm{Zn}$. The extraction of $\mathrm{Zn}, \mathrm{Cd}, \mathrm{Pb}, \mathrm{Cr}$ and Ni from the soil was accomplished using the DTPA-TEA pH 7,5 and Mehlich-1 solutions methods. The smallest $\mathrm{Zn}$ dose added to the soil as flue dust provided higher values than the critical levels for the aerial part of the plant. The $\mathrm{Pb}, \mathrm{Cd}, \mathrm{Cr}$ and Ni concentrations were, in the aerial part and in the soil, lower than the critical levels for toxicity. The correlation coefficients between the Zn extracted from the soil by the two solutions and Zn concentration in corn aerial part were significant and similar in both soils. It may be concluded, in the conditions studied, that flue dust is an efficient source of $\mathrm{Zn}$ to corn, and that the extractants were efficient to indicate its phytoavailability, but unefficient to the other metal studied. Zinc availability decreased with increasing $\mathrm{pH}$.

Key words: heavy metals, metallurgic residues, extractants.
\end{abstract}

( $\left.{ }^{1}\right)$ Trabalho financiado pela Fundação de Amparo à Pesquisa do Estado de São Paulo. Parte da tese de Mestrado da primeira autora. Recebido para publicação em 5 de dezembro de 2001 e aceito em 23 de setembro de 2002.

$\left({ }^{2}\right)$ Aluna de Mestrado do Instituto Agronômico (IAC), Caixa Postal 28, 13001-970 Campinas (SP). E-mail: gcsantos@esalq.usp.br.

$\left({ }^{3}\right)$ Centro de Pesquisa e Desenvolvimento de Solos e Recursos Ambientais, IAC.

(4) Bolsista da FAPESP.

$\left({ }^{5}\right)$ Com bolsa de produtividade científica do CNPq. 


\section{INTRODUÇÃO}

As indústrias siderúrgicas produzem vários resíduos que podem causar problemas ambientais, dependendo da forma e do local onde são descartados. Além disso, a disposição desses resíduos pode ser dispendiosa, elevando os custos da empresa. $\mathrm{O}$ aproveitamento de alguns desses materiais como fertilizante é uma forma de reduzir custos, bem como o impacto no ambiente (Guidi et al., 1988).

Muitos trabalhos têm mostrado o potencial de resíduos siderúrgicos como corretivos e fertilizantes (ACCIOly, 1996; AmAral-Sobrinho et al., 1997; Anderson e PARKPIAN, 1998; Silva, 1999). Dentre eles, destaca-se o pó-de-aciaria, obtido na produção do aço que contém aproximadamente $12 \%$ de $Z n$ e representa fonte potencial deste nutriente para as plantas. Entretanto, o pó-de-aciaria apresenta em sua constituição alguns metais pesados que podem provocar efeitos negativos em componentes bióticos e abióticos do ambiente, quando é utilizado na agricultura ou disposto de forma inadequada, pois pode resultar na transferência desses metais pesados do solo para as plantas e na sua entrada na cadeia alimentar em teores acima dos aceitáveis (Petruzzelli et al., 1989).

Entre os metais pesados que ocorrem nos resíduos, pode-se citar o $\mathrm{Cd}, \mathrm{Pb}, \mathrm{Cr}$ e $\mathrm{Ni}$, sendo suas concentrações dependentes do processo de geração de cada resíduo. Accioly (1996), utilizando pó-deaciaria contendo $1,3 \%$ de $\mathrm{Pb}$, e $0,01 \%$ de Cd, observou que os teores desses elementos no milho estavam abaixo dos limitesconsiderados tóxicos para a alimentação humana e animal.

Ressalta-se que, dependendo das características físicas e químicas do resíduo, a disponibilidade dos metais pesados nele contidos não é imediata, e sim gradual, liberando-os à medida que o resíduo vai reagindo com os constituintes do solo. As propriedades físicas e químicas do solo também são importantes por influenciarem a disponibilidade dos metais para as plantas (MATOs et al., 1995). Muitos trabalhos têm demonstrado que a retenção e solubilidade dos metais pesados no solo são fortemente influenciadas pelo $\mathrm{pH}$ (HARTER, 1983; Cunha et al., 1994; Martinez e Motto, 2000; Alcântara e CAmargo, 2001; Dias et al., 2001), pela quantidade de metal no solo (BASTA e TABATABAI, 1992), pela CTC (ZIPER et al., 1988; CunHA et al., 1994), pela matéria orgânica (ELLIOT et al., 1986; LEWIS e RULE, 2001) e pela mineralogia do solo (ZIPER et al., 1988). $\mathrm{O} \mathrm{pH}$ possui forte influência na retenção de metais no solo, sendo esta maior em pH elevado (BASTA et al., 1993; Cunha et al., 1994; Martinez e Motto, 2000; Alcântara e CAMARgo, 2001; Dias et al., 2001).

A disponibilidade de metais pesados, incluindo $\mathrm{Pb}, \mathrm{Ni}, \mathrm{Cd}$ e $\mathrm{Cu}$ nos solos, decresceu rapidamente quando o $\mathrm{pH}$ do solo aumentou de quatro para seis (MAZur, 1997). A solubilidade do Zn no solo é altamente dependente do $\mathrm{pH}$, decrescendo cem vezes para cada aumento de uma unidade no $\mathrm{pH}$ (LINDSAY, 1972). Além disso, solos com teores elevados de argila e matéria orgânica, por apresentarem maior CTC, possuem maior capacidade de retenção de metais catiônicos que solos mais arenosos e com teores menores de matéria orgânica (SHumAN, 1985; CunHA et al., 1994; Matos et al., 1996; AmAral et al., 1996). A matéria orgânica, embora represente, em geral, menos de $5 \%$ dos componentes sólidos, é responsável por, aproximadamente, $30 \%$ a $65 \%$ da CTC dos solos minerais e mais de $50 \%$ da CTC dos solos arenosos e orgânicos, daí sua importância na retenção de metais no solo.

A disponibilidade do metal pode ser avaliada usando-se um extrator apropriado, no qual a quantidade extraída é correlacionada com a quantidade ou com a concentração do elemento nas plantas. É o sistema mais empregado nas pesquisas (ABreu et al., 2002). Os extratores podem ser classificados em diversas categorias, dentre elas, soluções ácidas e soluções complexantes.

Os extratores ácidos extraem os metais, principalmente, pela dissolução dos minerais de argila, sendo a quantidade dependente da concentração do ácido, do tempo de extração e da relação solo/solução. As soluções ácidas mais testadas para extração das formas disponíveis de metais para as plantas são: $\mathrm{HCl} 0,1$ mol. $\mathrm{L}^{-1} \mathrm{e}$ Mehlich1. As soluções quelantes combinam-se com o íon metálico em solução formando complexos solúveis, diminuindo a sua atividade iônica na solução do solo. Em conseqüência, os íons dessorvem da superfície do solo ou da fase sólida para restabelecer o equilíbrio das formas iônicas na solução (ABREu et al., 2002). Os agentes quelantes mais usados, visando à seleção de métodos químicos para avaliar a disponibilidade de metais em amostras de solos brasileiros são o EDTA e o DTPA pH 7,3 (BATAGLIA e RAIJ, 1994) sendo este o mais difundido (ABREU et al., 1995).

O DTPA foi desenvolvido, inicialmente, para avaliar a disponibilidade de $\mathrm{Cu}, \mathrm{Fe}, \mathrm{Mn}$, e $\mathrm{Zn}$, e o Mehlich-1, para verificar a disponibilidade de $\mathrm{P}$ e cátions trocáveis. Esses métodos, entretanto, tiveram seu uso ampliado e atualmente têm sido usados também para $\mathrm{Pb}, \mathrm{Cd}$ e Ni (KorCaK e FANNING, 1978; SCHARUER et al., 1980; SOLTANPOUR, 1991). Silva (1999) verificou que a solução de $\mathrm{HCl}$ 0,1 mol. $\mathrm{L}^{-1}$ mostrou- 
se eficiente para avaliar a disponibilidade do Zn em solos que receberam o pó-de-aciaria; ineficiente, porém para avaliar a disponibilidade do $\mathrm{Pb}$ e do $\mathrm{Cu}$. RiBEIRO-FILHO et al. (2001), trabalhando com as soluções de DTPA e Mehlich-1, observaram que ambas foram adequadas para avaliar a disponibilidade de $\mathrm{Cd}, \mathrm{Ni}$, $\mathrm{Zn}$ e $\mathrm{Pb}$. Resultados semelhantes foram observados por Abreu et al. (1998) para Pb extraído com DTPA e Mehlich-3. Accioly (1996), ao avaliar o efeito da aplicação de um pó-de-aciaria ao solo, constatou que os extratores Mehlich-1 e DTPA não foram eficientes em extrair o $\mathrm{Pb}$ e o $\mathrm{Cd}$ do solo, sendo apenas para o $\mathrm{Zn}$. Percebe-se que para os metais $\mathrm{Cd}, \mathrm{Pb}, \mathrm{Cr}$ e Ni ainda não há uma definição clara de pesquisa sobre quais métodos usar. No Brasil, os resultados obtidos relativo ao emprego de diferentes extratores de metais pesados do solo são bastante contraditórios, em especial, aqueles referentes à disponibilidade de $\mathrm{Pb}, \mathrm{Cd}, \mathrm{Cr}$ e $\mathrm{Ni}$, metais freqüentemente encontrados em resíduos siderúrgicos (ABREu et al., 1995).

Tendo em vista o potencial de uso de pó-de-aciaria como fonte de zinco para as plantas, a possibilidade de acúmulo de metais não desejados no solo por esse material, sua disponibilidade para as plantas e a sua entrada na cadeia alimentar, deve-se investigar a utilização agronômica desse resíduo. Por isso, o objetivo deste trabalho foi avaliar a disponibilidade de zinco para o milho e o acúmulo de metais pesados no solo e na planta pelo uso do pó-de-aciaria.

\section{MATERIAL E MÉTODOS}

O experimento foi realizado em casa de vegetação, em Campinas (SP), de novembro de 2000 a janeiro de 2001, utilizando-se amostras superficiais $(0-20 \mathrm{~cm}$ de profundidade) de dois Latossolos Vermelhos, coletadas nos municípios de Rio das Pedras e Jaboticabal (SP), identificadas e aqui denominadas LVd-RP e LVd-J respectivamente. Os solos apresentaram as seguintes características: matéria orgânica $=33$ e $27 \mathrm{~g} \cdot \mathrm{dm}^{-3}$; $\mathrm{pH}$ em $\mathrm{CaCl}_{2} 0,01 \mathrm{~mol} \cdot \mathrm{L}^{-1}=$ 4,1 e 4,3; argila $=320$ e 250 g. $\mathrm{kg}^{-1}$; areia fina $=260$ e 340 g. kg-1 e areia grossa 170 e 310 g. $\mathrm{kg}^{-1}$, respectivamente, para LVd-RP e LVd-J.

O resíduo utilizado, proveniente de uma indústria paulista produtora de aço, apresentou, além da granulometria muito fina, em forma de pó, os seguintes teores de metais extraídos pelo método da água-régia (ABREU et al., 1996a): $\mathrm{Fe}=684 \mathrm{~g}^{\mathrm{kg}} \mathrm{kg}^{-1} ; \mathrm{Zn}=$ 122 g.kg $; \mathrm{Mn}=63$ g. $\mathrm{kg}^{-1} ; \mathrm{Pb}=24$ g.kg- ${ }^{-1} ; \mathrm{Cr}=6,0$ g.kg- $; \mathrm{Al}=3,5$ g.kg-1 $; \mathrm{Cu}=2,3$ g.kg ${ }^{-1} ; \mathrm{Ni}=0,48$ g.kge Cd $=0,13$ g. $\mathrm{kg}^{-1}$, apresentando, também, $\mathrm{pH} \mathrm{CaCl}_{2}$ $=9,4$ e $\mathrm{pH} \mathrm{H}_{2} \mathrm{O}=9,6$.
As amostras de solo foram secas, peneiradas e, seqüencialmente, aplicaram-se $\mathrm{CaCO}_{3}$ e $\mathrm{MgO}$ p.a. na relação 4:1 de $\mathrm{Ca} / \mathrm{Mg}$ na quantidade necessária para elevar o $\mathrm{pH}$ em $\mathrm{CaCl}_{2} 0,01 \mathrm{~mol} . \mathrm{L}^{-1}$ de cada amostra de solo a 5,0 e a 6,0, segundo curva de incubação. O período de incubação foi de 15 dias, mantendo-se a umidade próxima à capacidade de campo. Decorrido esse período, realizou-se a adubação básica misturando-se ao solo, 300 $\mathrm{mg} \cdot \mathrm{dm}^{-3} \mathrm{de}$ fósforo, na forma de superfosfato triplo granulado (passado em peneira de $2 \mathrm{~mm}$ ). Além do $\mathrm{P}$, misturou-se às amostras uma solução contendo o equivalente a, em mg.dm ${ }^{-3}, 60$ de N, 100 de $\mathrm{K}, 30$ de $\mathrm{S}, 1$ de $\mathrm{Cu}$ e 0,5 de B. Utilizaram-se como fonte desses elementos o nitrato de potássio, sulfato de amônio, ácido bórico e sulfato de cobre. Todos os reagentes utilizados foram de grau analítico.

O delineamento experimental foi inteiramente casualisado, em esquema fatorial, 2 (produtos) $x$ 4 (doses) $\times 2(\mathrm{pH}) \times 2$ (solos), com três repetições. Os produtos foram o pó-de-aciaria e o sulfato de zinco p.a., aplicados nas doses 0, 5, 50 e $150 \mathrm{mg}^{-\mathrm{dm}^{-3}}$ de Zn.

Decorridos sete dias da aplicação dos tratamentos, retirou-se uma subamostra de $100 \mathrm{~cm}^{3}$ de solo de cada vaso de plástico, com capacidade de $2 \mathrm{~L}$, a fim de determinar os teores de $\mathrm{Zn}, \mathrm{Pb}$, $\mathrm{Cd}, \mathrm{Cr}$ e Ni. Os extratores utilizados foram: (a) DTPA (LindSAY e NoRvell, 1978) e (b) Mehlich-1 (MEHLICH, 1953). As relações solo:solução foram de 1:2 (DTPA) e 1:10 (Mehlich-1). Determinaram-se os teores dos metais nos extratos por espectrometria de emissão atômica com plasma de argônio (ICP-AES).

Procedeu-se a semeadura do milho, híbrido BR 201, utilizando-se dez sementes por vaso, deixando-se, depois do desbaste, cinco plantas. Após o desbaste, em intervalos de cinco dias, adicionaram-se $20 \mathrm{~mL}$ por vaso de uma solução contendo $50 \mathrm{mg}$. $\mathrm{dm}^{-3}$ de $\mathrm{N}$ por aplicação. Usaramse como fontes o nitrato de cálcio e o de sulfato de amônio de forma alternada.

Realizou-se o corte da parte aérea aos 50 dias da emergência, sendo o material seco em estufa com circulação forçada de ar a $70^{\circ} \mathrm{C}$ até peso constante, moído e submetido à digestão via úmida, em forno microondas, de acordo com ABREU (1997).

Os dados foram submetidos à análise de regressão simples entre os teores de metais na parte aérea do milho e os extraídos dos solos pelos dois extratores. O programa de análise estatística empregado foi o MINITAB versão 13.0. 


\section{RESULTADOS E DISCUSSÃO}

A produção de matéria seca da parte aérea do milho não foi influenciada significativamente pela aplicação de doses crescentes de Zn ao solo (dados não apresentados), independentemente da fonte ou do solo, indicando que o teor natural de $\mathrm{Zn}$ dos solos era suficiente para atender a demanda das plantas. Resultado semelhante foi relatado por ACCIOLY (1996) em experimento com pó-de-aciaria e plantas de milho e alface.

Como a concentração e o acúmulo de Zn na matéria seca da parte aérea do milho revelaram comportamentos semelhantes, optou-se por apresentar somente a concentração de $\mathrm{Zn}$ na parte aérea. Os teores de $\mathrm{Zn}$ na parte aérea do milho variaram de 14,4 a 522,8 mg.kg-1 no LVd-J e de 18,1 a 267,5 mg. kg-1 no LVd-RP, ultrapassando o teor considerado como tóxico, 95 mg. $\mathrm{kg}^{-1}$ (GUPTA e GUPTA, 1998), nas doses mais altas das duas fontes. Resultados semelhantes foram observados por SiLva (1999) e AcCioly (1996). Ressalta-se que na avaliação visual das plantas, aos 40 dias da emergência, notou-se o amarelecimento das folhas mais novas naqueles tratamentos que receberam $150 \mathrm{mg} . \mathrm{dm}^{-3}$ de $\mathrm{Zn}$, independentemente do solo ou do $\mathrm{pH}$, o que pode ser decorrente da toxicidade de $\mathrm{Zn}$ (ADRIANO et al., 1971).

Para ambas as fontes testadas, o aumento da dose de zinco foi acompanhado pelo aumento da concentração do nutriente na parte aérea das plantas de milho, sendo a relação linear e significativa (Figura 1). Entretanto, o pó-de-aciaria proporcionou teores de Zn na parte aérea do milho menores que o sulfato de zinco, discordando dos resultados apresentados por AcCioly (1996) e SILVA (1999) que encontraram teores de zinco na parte aérea maiores nos tratamentos com pó-de-aciaria que nos tratamentos com sulfato de zinco.

No LVd-J, com a aplicação de $50 \mathrm{mg} \cdot \mathrm{dm}^{-3}$ de $\mathrm{Zn}$ ao solo, foram obtidos 78,93 e 151,89 $\mathrm{mg}$ de $\mathrm{Zn}$ por quilo de matéria seca, respectivamente, para o póde-aciaria e para o sulfato de zinco; a mesma tendência foi observada para o LVd-RP. Ressalta-se que mesmo usando a menor dose $\left(5 \mathrm{mg} \cdot \mathrm{dm}^{-3}\right)$, esta foi suficiente para proporcionar teores de $\mathrm{Zn}$ na parte aérea acima do nível crítico para plantas de milho que está em torno de $15 \mathrm{mg} . \mathrm{kg}^{-1}$ (TERMAN et al., 1972). Esses resultados evidenciaram que, apesar de o póde-aciaria ser capaz de suprir a planta em Zn, a dose necessária será maior que aquela recomendada para o sulfato de zinco, graças à sua menor solubilidade. As maiores concentrações de $\mathrm{Zn}$ na parte aérea do milho ocorreram quando as plantas foram cultivadas no LVd-J (Figura 1). Essa diferença pode ser em virtude das características físico-químicas dos solos,

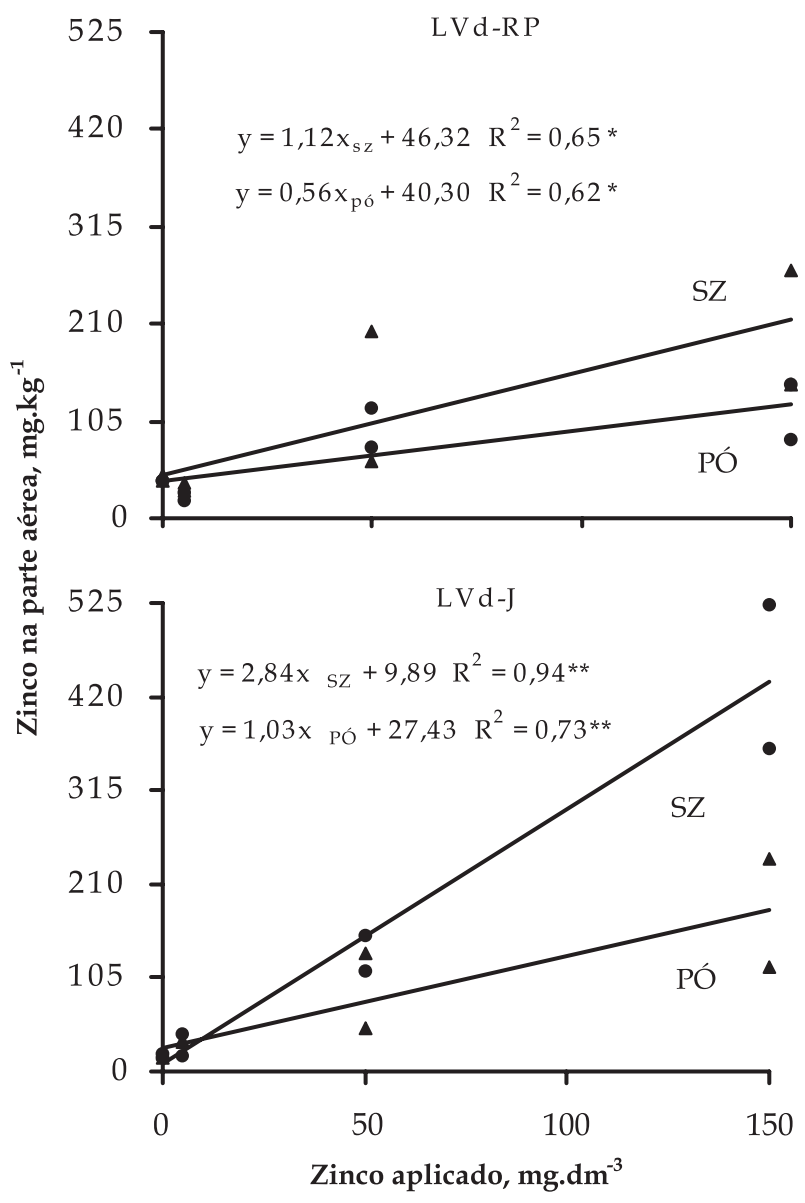

Figura 1. Concentração de zinco na parte aérea do milho, influenciada por doses de zinco supridas via sulfato de zinco (SZ) e pó-de-aciaria (PÓ) a dois latossolos. * **: Significativos ao nível de $5 \%$ e $1 \%$ respectivamente.

uma vez que o LVd-RP apresentou teores de argila e matéria orgânica maiores que o LVd-J, o que pode ter proporcionado maior retenção de zinco nesse solo. De acordo com alguns autores, solos com teores elevados de argila e matéria orgânica, por apresentarem maior CTC, possuem maior capacidade de retenção de zinco que solos mais arenosos e com menores teores de matéria orgânica (CunHa et al., 1994; Matos et al., 1996).

Os teores de $\mathrm{Cd}, \mathrm{Cr}$ e Ni na matéria seca do milho apresentaram, respectivamente, as seguintes amplitudes: 0,01 a 0,18; 0,04 a 2,24 e 0,01 a 1,24 mg.kg${ }^{1}$ e estão abaixo dos níveis considerados críticos para toxicidade. De acordo com McNichOL e BECKETT (1985), teores acima de $8 \mathrm{mg} . \mathrm{kg}^{-1} \mathrm{de} \mathrm{Ni}, 4 \mathrm{mg} \cdot \mathrm{kg}^{-1}$ de $\mathrm{Cd} \mathrm{e} 2 \mathrm{mg} \cdot \mathrm{kg}^{-1}$ de $\mathrm{Cr}$ podem ocasionar toxicidade em muitas plantas, diminuindo a produção. A amplitude de variação dos teores de $\mathrm{Pb}$ na parte aérea do milho foi ligeiramente maior que as verificadas 
para $\mathrm{Ni}, \mathrm{Cr}$ e Cd, sendo de 0,01 a 6,1 mg.kg-1. Essas concentrações estão abaixo daquelas consideradas como tóxicas para o milho, 15 mg.kg-1 (GRÜN et al., 1985) e $30 \mathrm{mg} . \mathrm{kg}^{-1}$ (Kabata-Pendias e Pendias, 1992). Entretanto, comparando-se a concentração de $\mathrm{Pb}$ na parte aérea do milho cultivado em solo que não recebeu adição de $\mathrm{Zn}$ com a concentração de $\mathrm{Pb}$ no milho que recebeu $150 \mathrm{mg} \cdot \mathrm{dm}^{-3}$ de $\mathrm{Zn}$ sob a forma de pó-de-aciaria, observa-se que houve, em média, acréscimo de 1,0 e 5,0 mg. $\mathrm{kg}^{-1}$ na concentração de $\mathrm{Pb}$ na parte aérea do milho, respectivamente, para o LVd-J e LVd-RP. Embora tenha sido observada variação nos teores de $\mathrm{Cd}, \mathrm{Cr}, \mathrm{Ni}$ e $\mathrm{Pb}$ na parte aérea do milho, não houve efeito significativo da dose do pó-de-aciaria na concentração desses metais com exceção para o $\mathrm{Pb}$ e Ni. Resultado semelhante foi observado por Silva (1999) em experimento com póde-aciaria em plantas de feijão.

Os teores de $\mathrm{Zn}$ nos solos apresentaram amplitude de 0,8 a $94,4 \mathrm{mg} \cdot \mathrm{dm}^{-3}$ (DTPA) e 2,3 a 109,3 mg.dm ${ }^{-3}$ (Mehlich-1) (Figura 2). Cabe destacar que, mesmo tendo sido observados sintomas visuais de toxicidade de $\mathrm{Zn}$ nas plantas, os teores do elemento no solo foram inferiores ao teor sugerido como tóxico, 130 mg.kg-1 ${ }^{-1}$ conforme INTER-DEPARTMENTAL COMMITTEE ON THE REDEVELOPMENT OF CONTAMINATED LAND (1987). É importante mencionar que este teor sugerido como tóxico foi obtido com solução de EDTA 0,05 mol. $\mathrm{L}^{-1}$, enquanto neste experimento utilizaram-se as soluções de DTPA e Mehlich-1 para extraí-lo do solo.

Considerando todos os tratamentos, os teores de $\mathrm{Pb}$ no solo variaram de 0,23 a $7,23 \mathrm{mg} \cdot \mathrm{dm}^{-3}$ (DTPA) e 0,01 a $7,7 \mathrm{mg} \cdot \mathrm{dm}^{-3}$ (Mehlich-1). Esses resultados estão acima dos teores encontrados por ABREU et al. (1995), em amostras superficiais de solos do Estado de São Paulo, que observaram amplitude de variação para o $\mathrm{Pb}$ de 0,8 a $5,9 \mathrm{mg} \cdot \mathrm{dm}^{-3}$ (DTPA) e 1,1 a 2,6 mg.dm ${ }^{-3}$ (Mehlich-1). Entretanto, estão abaixo de 20 $\mathrm{mg} . \mathrm{dm}^{-3}$ de $\mathrm{Pb}$ (DTPA), considerado como elevado por Wallace e Wallace (1994).

Apesar de o $\mathrm{Cd}, \mathrm{Cr}$ e $\mathrm{Ni}$ estarem presentes na constituição química do pó-de-aciaria, a análise de solo por DTPA indicou a presença desses metais em baixas concentrações, 0,01 a 0,$15 ; 0,01$ a 0,29 e 0,01 a $0,25 \mathrm{mg} \cdot \mathrm{dm}^{-3}$, sendo impossível detectar esses metais quando se utilizou a solução de Mehlich-1,

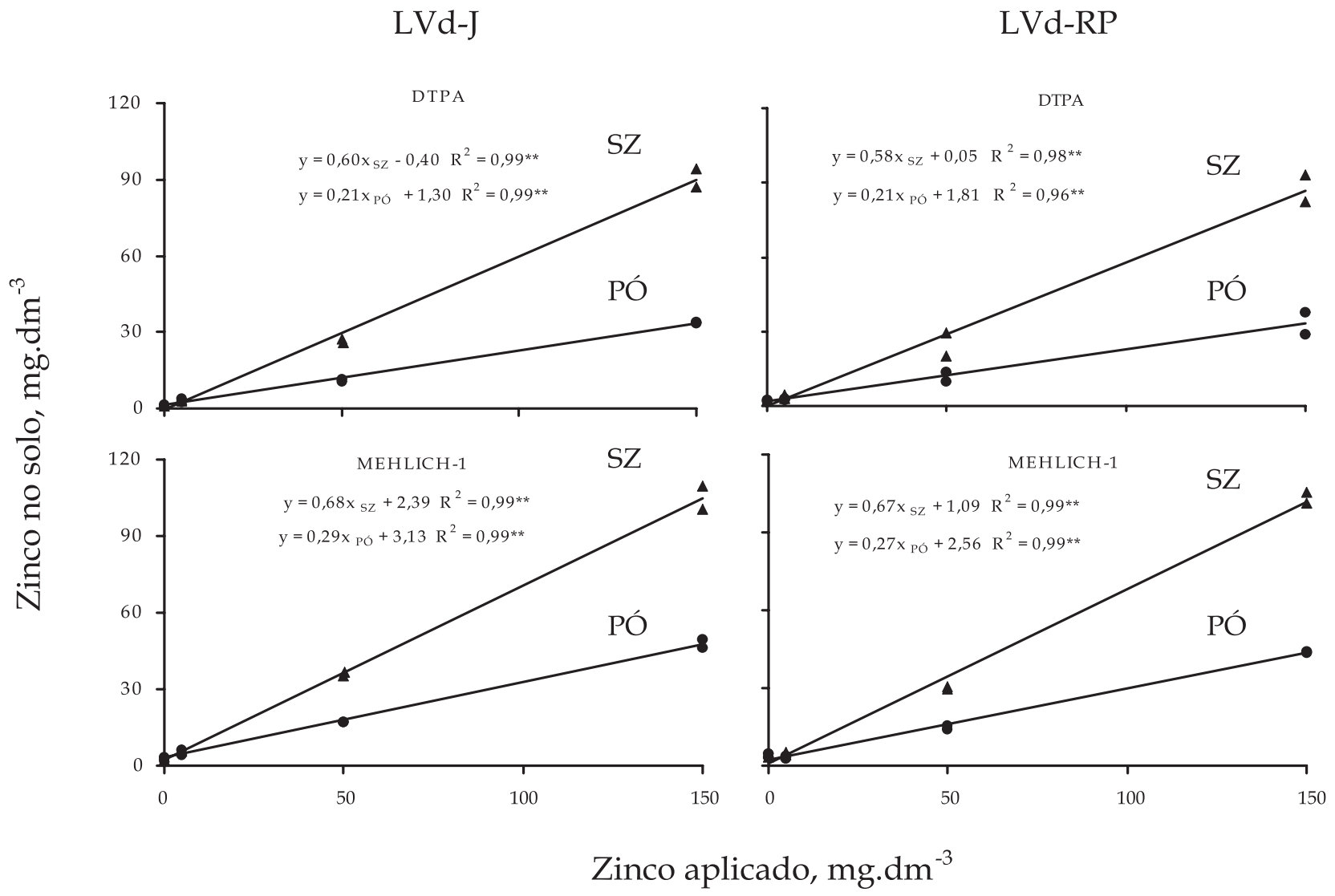

Figura 2. Teores de zinco extraídos pelas soluções de DTPA e Mehlich-1 influenciados por doses de zinco supridas via pó-de-aciaria (PÓ) e sulfato de zinco (SZ) a dois latossolos. **: Significativo ao nível de $1 \%$. 
LVd-J

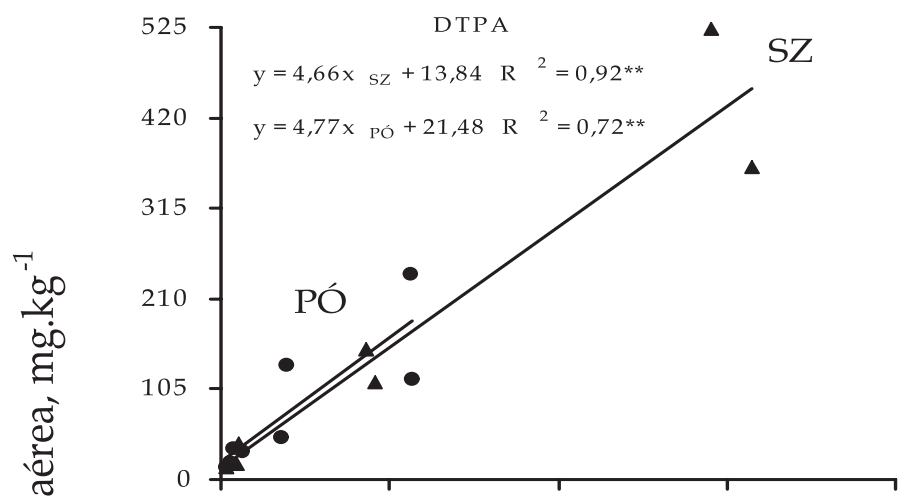

\section{MEHLICH-1}

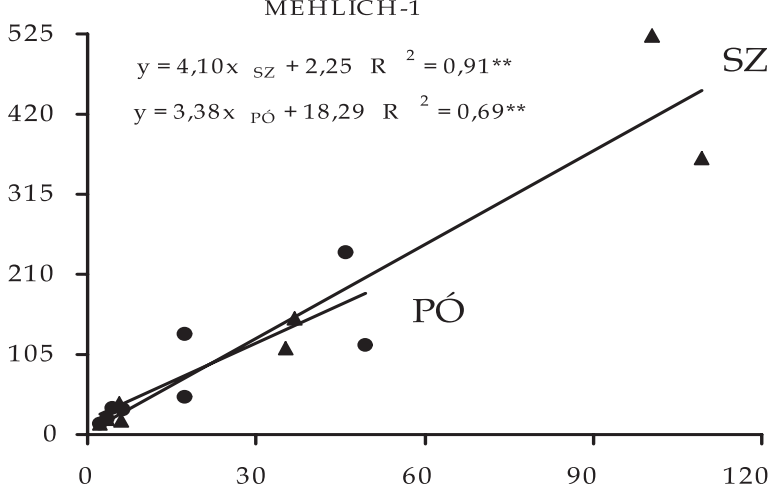

LVd-RP

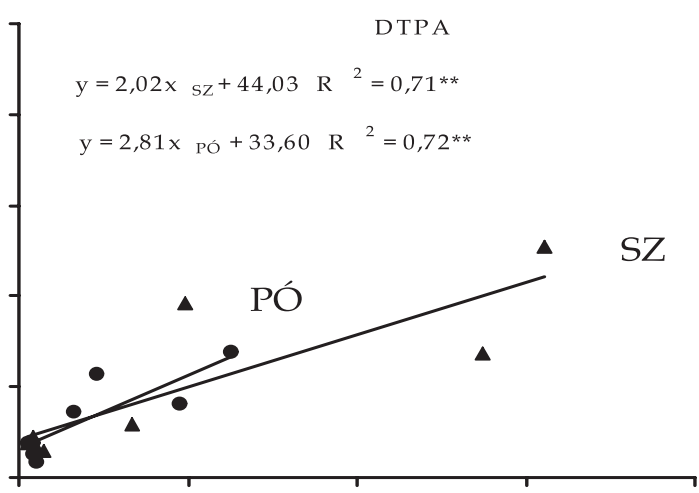

MEHLICH-1

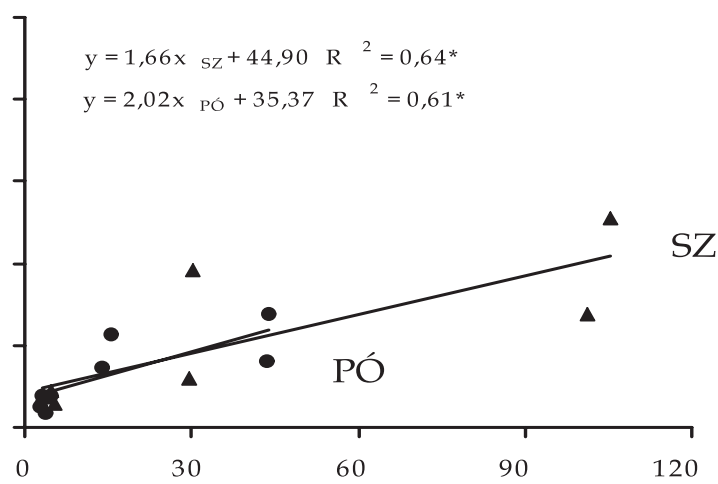

Zinco extraído do solo, mg.dm ${ }^{-3}$

Figura 3. Relação entre os teores de zinco extraídos dos latossolos pelas soluções de DTPA e Mehlich-1 e a concentração do elemento na matéria seca da parte aérea do milho. * ${ }^{* *}$ : Significativos ao nível de $5 \%$ e $1 \%$ respectivamente.

concordando com Accioly (1996) e Silva (1999). Esses resultados estão de acordo, também, com os de ABREU et al. (2001) ao analisarem 5.614 amostras do Estado de São Paulo, cujos teores máximos observados de $\mathrm{Cd}, \mathrm{Cr}$, e $\mathrm{Ni}$ foram 1,$0 ; 0,0$ e $1,1 \mathrm{mg}^{\mathrm{dm}} \mathrm{dm}^{-3}$ respectivamente. Ressalta-se que os teores de $\mathrm{Cr}$ e $\mathrm{Ni}$ foram inferiores aos níveis considerados como tóxicos, 20 e 25 mg. $\mathrm{kg}^{-1}$ extraídos com EDTA 0,05 mol.L - $^{-1}$ (InTER-Departmental COMMITTEe ON THE REDEVELLPMENT OF CONTAMINATED LAND, 1987) e o teor de Cd foi inferior a 3,0 mg. $\mathrm{kg}^{-1}$. Segundo Mengel e KIRKBY (1987) solos com teores de Cd acima desse valor são impróprios para o cultivo agrícola.

É interessante observar o comportamento diferencial dos extratores DTPA e Mehlich-1, usados para avaliar a disponibilidade de Zn às plantas. Verifica-se que o Mehlich-1 apresentou maior capacidade de extração que o DTPA, independentemente do tratamento aplicado (Figura 2). A maior extração do Mehlich-1 deve-se à sua elevada acidez que, provavelmente, solubilizou parte do $\mathrm{Zn}$ adsorvido aos óxidos, enquanto o DTPA 7,3 , por ser um extrator alcalino, não conseguiu fazê-lo. Resultados semelhantes foram observados por Ribeiro-FilHo et al. (1999).

Os extratores foram eficientes em avaliar a disponibilidade de zinco para plantas de milho (Figura 3). Resultado semelhante foi relatado por AcCiOly (1996) em experimento com pó-de-aciaria em plantas de milho e alface.

Os coeficientes de correlação entre o Zn extraído do solo pelas soluções de DTPA e Mehlich-1 e a sua concentração na parte aérea do milho foram menores para o póde-aciaria que para osulfato de zinco, comexceção doDTPA no LVd-RP, que apresentou 0,71 e 0,72 como coeficientes de correlação para o sulfato de zinco e para o pó-de-aciaria (Figura 3). Para o LVd-J os coeficientes de determinação foram: 0,92 e 0,72 (DTPA) e 0,91 e 0,69 (Mehlich-1), respectivamente, para o sulfato de zinco e pó-deaciaria, sendo observado efeito similar para o LVd-RP. 


\section{LVd-J}

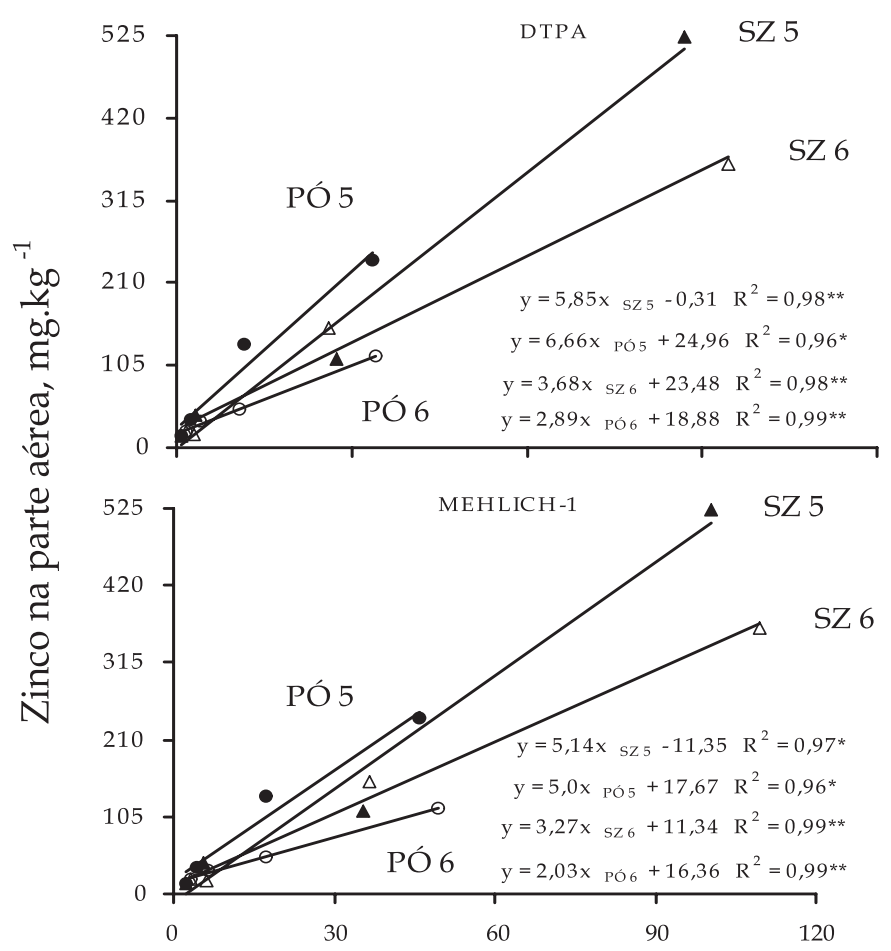

LVd-RP
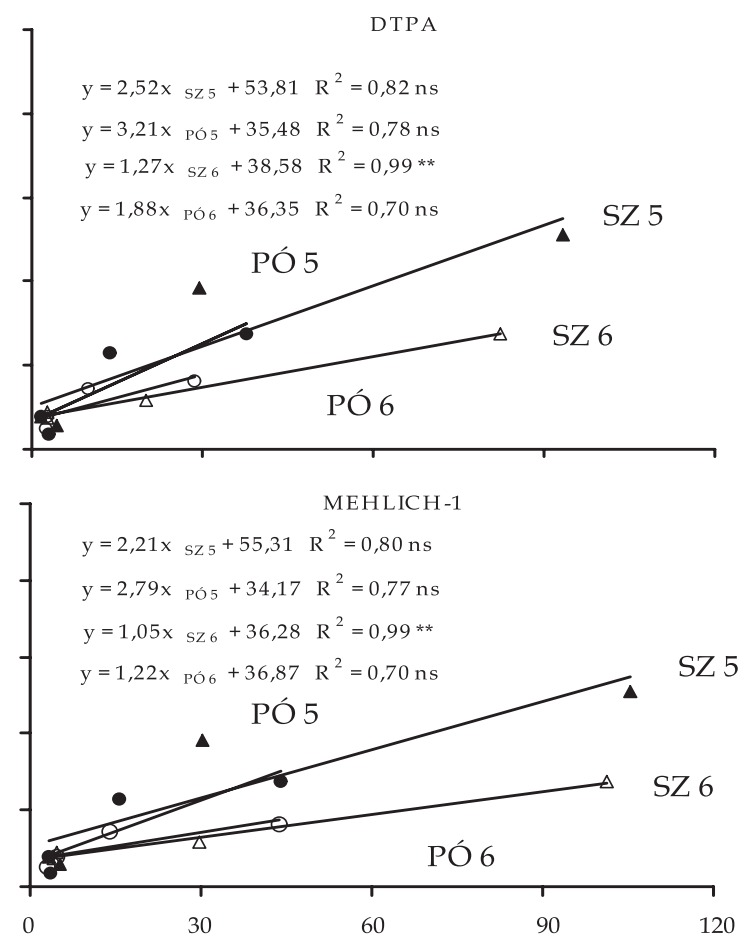

Zinco extraído do solo, mg.dm ${ }^{-3}$

Figura 4. Relação entre os teores de zinco extraídos dos dois latossolos pelas soluções de DTPA e Mehlich-1 e a concentração do elemento na matéria seca da parte aérea do milho em dois diferentes valores de pH (5 e 6). ns, * ,*: Não significativo e significativos ao nível de $5 \%$ e $1 \%$ respectivamente.

Em uma avaliação mais detalhada do comportamento dos extratores em relação à mudança de $\mathrm{pH}$, observa-se que, independentemente da fonte de zinco utilizada, os extratores foram eficientes em avaliar a disponibilidade do Zn apenas no LVd-J, não o sendo para o LVd-RP, a menos quando se utilizou o sulfato de zinco a pH 6 (Figura 4).

Por meio da análise de regressão linear, realizada entre o teor de $\mathrm{Zn}$ extraído do solo pelas soluções extratoras e a concentração do elemento na parte aérea do milho (Figura 4), independentemente da fonte utilizada, verifica-se diminuição na disponibilidade do elemento com o aumento do $\mathrm{pH}$. À medida que o $\mathrm{pH}$ aumentou de 5,0 para 6,0, houve diminuição dos teores de $\mathrm{Zn}$ na parte aérea do milho, independentemente do solo e da fonte utilizada. Com a elevação do $\mathrm{pH}$ ocorre a diminuição das formas livres de $\mathrm{Zn}^{2+}$ pela complexação do $\mathrm{Zn}$ com o $\mathrm{OH}^{-}$ (SHUMAN, 1986). Admitindo-se que a espécie livre $\mathrm{Zn}^{2+}$ é a forma predominantemente absorvida, sua diminuição explica a menor absorção de $\mathrm{Zn}$ pelas plantas (Machado e PAVAN, 1987).
Para ambos os solos verifica-se que o pó-de-aciaria proporcionou menores teores de zinco na parte aérea que o sulfato de zinco, o que pode estar relacionado à sua menor solubilidade em água quando comparado ao sulfato de zinco. Entretanto, também podem estar relacionadas com as diferenças nos constituintes adicionados com os metais, pois os sais metálicos adicionam apenas o ânion ligado ao metal enquanto o pó-de-aciaria adiciona óxidos metálicos. ABREU et al. (1996b) verificaram para o manganês, em plantas de soja, menor eficiência dos óxidos em relação ao sulfato.

Em geral, o Zn foi mais disponível no LVd-J que no LVd-RP (Figura 4). Essa tendência se deve às características físico-químicas dos solos. O LVd-J apresenta menor teor de argila e de matéria orgânica que o LVd-RP, que possibilita menor adsorção de $\mathrm{Zn}$ nos colóides do solo, tornando-o mais disponível às plantas, fato também registrado por Couto et al. (1985). Para Shuman (1975), solos com elevados conteúdos de matéria orgânica e argila possuem maior capacidade de adsorção e maior energia de 
ligação para adsorção de Zn, que os arenosos com pouca matéria orgânica e argila.

Quanto à capacidade dos extratores em avaliar a disponibilidade dos metais $\mathrm{Pb}, \mathrm{Cd}, \mathrm{Cr}$ e $\mathrm{Ni}$ para plantas de milho, verificou-se (dados não apresentados) que nenhum deles foi eficiente. Para os metais $\mathrm{Cd}, \mathrm{Cr}$ e $\mathrm{Ni}$ a falta de correlação pode ter ocorrido, entre outros fatores, em virtude das baixas concentrações nas amostras de solo. Já para o $\mathrm{Pb}$ a falta de correlação significativa pode ser decorrente da pequena variação dos teores do elemento no solo e na planta. Resultados semelhantes foram relatados por ABREU et al. (1995) ao avaliar a eficiência dos métodos DTPA e Mehlich-1 para metais pesados.

\section{CONCLUSÕES}

1. O pó-de-aciaria pode ser considerado potencial fonte de zinco para o milho, sem causar a contaminação do solo por metais pesados.

2. Os extratores DTPA e Mehlich-1 podem ser utilizados como métodos químicos para indicar a fitodisponibilidade de zinco em latossolos adubados com pó-de-aciaria.

\section{AGRADECIMENTOS}

À Fundação de Amparo à Pesquisa do Estado de São Paulo (FAPESP) pelo apoio financeiro.

\section{REFERÊNCIAS BIBLIOGRÁFICAS}

ABREU, C.A.; ABREU, M.F.; BERTON, R.S. Análise química de solos para metais pesados. In: TÓPICOS CIÊNCIAS DO SOLO. Viçosa: Sociedade Brasileira de Ciência do Solo, 2002. v.2, p.1-48.

ABREU, C.A.; ABREU, M.F.; RAIJ, B. van; SANTOS, W.R. Comparação de métodos de análise para avaliar a disponibilidade de metais pesados em solos. Revista Brasileira de Ciência do Solo, Campinas, v.19, p.463-468, 1995.

ABREU, C.A.; RAIJ, B. van.; TANAKA, R.T. Fontes de manganês para a soja na análise de solo. Revista Brasileira de Ciência do Solo, Campinas, v.20, p.91-97, 1996b.

ABREU, C.A.;SANTOS, W.R.; PAZGONZALEZ, A. Routine Soil Analysis as Diagnostic Criteria for Heavy Metals Contamination. In: INTERNATIONALCONFERENCE ON THE BIOGEOCHEMISTRY OF TRACE ELEMENTS, $6^{\text {th }}$ 2001. Guelph, Canada. (CD-Rom)

ABREU, C.A.; ABREU, M.F.; ANDRADE, J.C. Distribuição de chumbo no perfil de solo avaliada pelas soluções de DTPA e Mehlich-3. Bragantia, Campinas, v.57, n.1, p.185-192, 1998.
ABREU, M.F. Extração e determinação simultânea por emissão em plasma de nutrientes e elementos tóxicos em amostras de interesse agronômico. 1997, 135f. Dissertação (Doutorado em Química) - Universidade Estadual de Campinas.

ABREU, M.F.; ANDRADE, J.C. BERTON, R.S. Comparison of methods to evaluate heavy metal in organic wastes. Communications in Soil Science and Plant Analysis, New York, v.27, p.1125-1135, 1996a.

ACCIOLY, A.M.A. Pó de forno de siderurgia como fonte de micronutrientes e seu efeito no solo e na planta. 1996. 70f. Dissertação Mestrado (Solos e Nutrição de Plantas) Universidade Federal de Lavras.

ADRIANO, D.C.; PAULSEN, G.M.; MURPHY, L.S. Phosphorus-iron and phosphorus-zinc relationship in corn (Zea mays L.) seedlings as affected by mineral nutrition. Agronomy Journal, Madison, v.63, p.36-39, 1971.

ALCÂNTARA, M.A.K.; CAMARGO, O.A. Fator de retardamento e coeficiente de dispersão-difusão para crômio (III) em solos muito intemperizados, influenciados pelo $\mathrm{pH}$, textura e matéria orgânica. Revista Brasileira de Ciência do Solo,Viçosa, v.25, p.209216, 2001.

AMARAL-SOBRINHO, N.M.B.; VELLOSO, A.C.X.; OLIVEIRA, C. Solubilidade de metais pesados em solo tratado com resíduo siderúrgico. Revista Brasileira de Ciência do Solo, Campinas, v.21, n.1, p.9-16, 1997.

AMARAL, R.D.; BARROS, N.F.; COSTA, L.M.; FONTES, M.P.F. Efeito de um resíduo da indústria de zinco sobre a química de amostras de solo e plantas de milho. Revista Brasileira de Ciência do Solo, Campinas, v.20, p.433-440, 1996.

ANDERSON, W.B.; PARKPIAN, P. Effect of soil applied iron by product on micronutrient concentration in sorghum cultivars. Journal of Plant Nutrition, Lexington, v.7, p.1333-1343, 1998.

BASTA, N.T.; PANTONE, D.J.; TABATABAI, M.A. Path analysis of heavy metal adsorption by soil. Agronomy Journal, Madison, v.85, p.1054-1057, 1993.

BASTA, N.T.; TABATABAI, M.A. Effect of cropping systems on adsorption of metals by soils. II. Effect of pH. Soil Scence, Baltimore, v.153, p.195-204, 1992.

BATAGLIA, O.C.; RAIJ, B. van. Soluções extratoras na avaliação da fitodisponibilidade do zinco em solos. Revista Brasileira de Ciência do Solo, Campinas, v.18, p.457-461, 1994.

COUTO, C.; NOVAIS, R.F.; BARROS, N.F.; NEVES, J.C.L. Resposta do eucalipto à aplicação de zinco em mostras de solos de cerrado. Revista Árvore, Viçosa, v.9, p.134-148, 1985. 
CUNHA, R.C.A.; CAMARGO, O.A.; KINJO, T. Retenção de zinco em solos paulistas. Bragantia, Campinas, v.53, n.2, p.291-301, 1994.

DIAS, N.M.; ALLEONI, L.R.F.; CASAGRANDE, J.C.; CAMARGO, O.A. Adsorção de cádmio em dois latossolos ácricos e um nitossolo. Revista Brasileira de Ciência do Solo, Viçosa, v.25, p.297-304, 2001.

ELLIOT, H.A.; LIBERATI, M.R.; HUANG, C.P. Competitive adsorption of heavy metals by soils. Journal of Environmental Quality, Madison, v.15, p.214-219, 1986.

GRÜN, M.; KRONEMANN, H.; POEDLESAK, W.; MACHELETT, B. Blei in der Umwelt: Pflanze. Proceedings of Mengen-und Spurenelemente Arbeitst. Leipzig: Karl-Marx University, 1985. p.201-215.

GUIDI, G.; PERA, A.; GIOVANNETTI, M.; POGGIO, G.; De BERTOLDI, M. Variations of soil structure and microbial population in a compost amended soil. Plant and Soil, Dordrecht, v.106, p.113-119, 1988.

GUPTA, U.C.; GUPTA, S.C. Trace element toxicity relationship to crop production and livestock and human health: implications for management. Communications in Soil Science and Plant Analysis, New York, v.29, p.1491-1522, 1998.

HARTER, R.D. Effect of soil pH on adsorption of lead, copper, zinc, and nickel. Soil Science Society of American Journal, Madison, v.47, p.47-51, 1983.

INTER-DEPARTMENTAL COMMITTEE ON THE REDEVELOPMENT OF CONTAMINATED LAND. Guindance on the assessment and redevelopment of contaminated land. 59/83. 2.ed. London: Department of the environment, 1987.

KABATA-PENDIAS, A.; PENDIAS, H. Trace elements in soil and plants. 2.ed. Boca Raton: CRC Press, 1992. 365p.

KORCAK, R.F.; FANNING, D.S. Extractability of cadmium, copper, nickel, and zinc by double acid versus DTPA and plant content at excessive soil levels. Journal of Environmental Quality, Madison, v.7, p.506-512, 1978.

LEWIS, S.E.; RULE, J.H. Effects of organic matter and iron oxides on mobility of trace metals from flue dust contaminated soil. In: INTERNATIONAL CONFERENCE ON THE BIOGEOCHEMISTRY OF TRACE ELEMENTS, $6^{\text {th }}, 2001$, Guelph. (CD-Rom).

LINDSAY, W.L. Inorganic phase equilibria of micronutrients in soil. In: MORTVEDT, J.J. (Ed.). Micronutrients in agriculture. Madison: Soil Sciece Society of America, 1972. p.41-57.

LINDSAY, W.L.; NORVELL, W. Development of DTPA soil for zinc, iron, manganese and copper. Soil Science Society of American Journal, Madison, v.42, p.421-428, 1978.
MACHADO, P.L.O.; PAVAN, M.A. A adsorsão de zinco por alguns solos do Paraná. Revista Brasileira de Ciência do Solo, Campinas, v.11, p.253-256, 1987.

MARTINEZ, C.E.; MOTTO, H.L. Solubility of lead, zinc and copper added to mineral soils. Environmental Pollution, Barking Essex, v.107, p.153-158, 2000.

MATOS, A.T.; FONTES, M.P.F.; JORDÃO, C.P.; COSTA, L.M. Mobilidade e formas de retenção de metais pesados em Latossolo Vermelho-Amarelo. Revista Brasileira de Ciência do Solo, v.20, p.379-386, 1996.

MATOS, A.T.; FONTES, M.P.F.; COSTA, L.M.; MARTINEZ, J.A. Fatores de retardamento e coeficientes de dispersão-difusão dos metais zinco, cádmio, cobre e chumbo em solos do município de Viçosa-MG. II. Correlação com algumas propriedades físicas dos solos. In: CONGRESSO BRASILEIRO DE CIÊNCIA DOSOLO, 25., 1995, Viçosa. Anais... Viçosa: Sociedade Brasileira de Ciência do Solo/UFV, 1995. p.2333-2336.

MAZUR, N. Níquel, chumbo, zinco e cobre em solos que receberam composto de resíduos sólidos urbanos. 1997, 129f. Tese (Doutorado em Solos e Nutrição de Plantas) - Universidade Federal de Viçosa.

McNICHOL, R.D.; BECKETT, P.H.T. Plant and Soil, Dordrecht, v.85, p.107-129, 1985.

MEHLICH, A. Determination of $\mathrm{Ca}, \mathrm{Mg}, \mathrm{K}, \mathrm{Na}$ e $\mathrm{NH}_{4}$. Raleigh: North Carolina Soil Test Divion, 1953.

MENGEL, K.; KIRKBY, E.A. Principles of plant nutrition. 4.ed. Bern: International Potash Institute, 1987. 687p.

PETRUZZELLI, G.; LUBRANO, L.; GUIDI. G. Uptake by corn and chemical extractability of heavy metals from a four year compost treated soil. Plant and Soil, Dordrecht, v.116, p.23-27, 1989.

RIBEIRO-FILHO, M.R.; CURI, N.; SIQUEIRA, J.O.; MOTTA, P.E.F. Metais pesados em solos de área de rejeitos de industria de processamento de zinco. Revista Brasileira de Ciência do Solo, Viçosa, v.23, p.453464, 1999.

RIBEIRO-FILHO, M.R.; SIQUEIRA, J.O.; CURI, N.;SIMÃO, J.B.P. Fracionamento e biodisponibilidade de metais pesados em solo contaminado, incubado com materiais orgânicos e inorgânicos. Revista Brasileira de Ciência do Solo, Viçosa, v.25, p.495-507, 2001.

SCHARUER, P.S.; WRIGHT, W.R.; PELCHAT, J. Sludgeborne heavy metal availability and uptake by vegetable crops under field conditions. Journal of Environmental Quality, Madison, v.9, p.69-73, 1980.

SHUMAN, L.M. Effect of ionic strength and anions on zinc adsorption by two soils. Soil Science Society of America Journal, Madison, v.50, p.1438-1442, 1986. 
SHUMAN, L.M. Fractionation method for soil microelements. Soil Science, Baltimore, v.140, p.10-12, 1985.

SHUMAN, L.M. The effect of soil properties on zinc adsorption by soils. Proceedings Soil Science Society of America, Madison, v.39, p.454-458, 1975.

SILVA, F.A.M. Fracionamento e biodisponibilidade de metais para o feijoeiro em solos tratados com pó de forno de aciaria elétrica. 1999. 80f. Dissertação (Mestrado) Universidade Federal de Lavras.

SOLTANPOUR, P.N. Determination of nutrient availability and elemental toxicity by AB-DTPA soil test and ICPS. Advances in Soil Science, Baltimore, v.16, p.165-190, 1991.
TERMAN, G.L.; GIORDANO, P.M.; ALLEN, S.E. Relationships between dry matter yelds and concentration of $\mathrm{Zn}$ and $\mathrm{P}$ in young corn plants. Agronomy Journal, Madison, v.64, n.4, p.684-687, 1972.

WALLACE, G.A.; WALLACE, A. Lead and other potenctially toxic heavy metals in soil. Communications in Soil Science and Plant Analysis, New York, v.25, p.137-141, 1994.

ZIPER, C.; KOMARNENI, S.; BAKER, D.E. Specific cadmium sorption in relation to the crystal chemistry of clay minerals. Soil Science Society of America Journal, Madison, v.52, p.49-53, 1988. 